levels of SP-D increased not only in GGO dominant type but in PCO dominant type (Fig. 2). The extent of honeycombing did not correlatewith the levels of SP-A or SP-D. The levels of SP-D in serum rather than SP-A might predict the progression from alveolar collapse to fibrosis (4).

The relationship between the rate of deterioration in the pulmonary function test (\% TLC and \%VC) and the serum levels of SP-A and SP-D are shown in Figs. 3 and 4. The mean follow-up interval of patients IIP was 35 months. The levels of SP-A and SP-D at the start of follow-up did not correlate with $\% \mathrm{TLC}$ and/or \%VC. The serum level of SP-A, obtained during the initial visit, did not significantly correlate with the rate of deterioration in the pulmonary fuction test. However, the level of SP-D in serum was significantly correlated with the rate of the deterioration of \% TLC and/or \% VC. The IIP patients with higher levels of SP-D in serum may have a greater chance of falling into restrictive pulmonary dysfunction more rapidly than those with lower levels of SP-D.

We investigated whether there is a relationship between the prognosis and serum marker levels in patients with IIP. Ten patients died of acute exacerbation within 3 years of followup. All 10 nonsurvivors compared with the IIP patients who were still alive after 3 years exhibited significantly higher levels than the cut-off point of SP-A, SP-D and KL-6 (Fig. 5).
The patients with IIP with less than cut-off levels of the three serum markers at the initial visit were still surviving beyond 3 years.

In conclusion, the new serum biomarkers, SP-A, SP-D, KL6 may be useful to understand the clinical characteristics, especially to predict disease activity, prognosis and survival time in patients with IIP. Thus, it appears to be possible to utilize the combination assay of three serum biomarkers as a very useful marker for clinical investigation of patients with diffuse interstitial pneumonia.

\section{References}

1) Kohno N, Kyoizumi S, Awaya Y, Fukuhara H, Yamakido M, Akiyama M. New serum indicator of interstitial pneumonitis activity: Sialylated carbohydrate antigen KL-6. Chest 96: 68-73, 1989.

2) Kuroki Y, Tsutahara $S$, Shijubo N, et al. Elevated levels of lung surfactant protein $\mathrm{A}$ in sera from patients with idiopathic pulmonary fibrosis and pulmonary alveolar proteinosis. Am Rev Respir Dis 147: 723-729, 1993.

3) Honda $Y$, Kuroki $Y$, Matsuura E, et al. Pulmonary surfactant protein $D$ in sera and bronchoalveolar lavage fluids. Am J Respir Crit Care Med 152: 1860-1866, 1995.

4) Takahashi H, Fujishima T, Koba H, et al. Serum surfactant protein A and $\mathrm{D}$ as prognostic factors in idiopathic pulmonary fibrosis and their relationship to disease extent. Am J Respir Crit Care Med 162: 1109-1114, 2000.

\title{
2. Obstructive Lung Disease
}

\author{
Ken ОнтА \\ Professor of Medicine, Department of Medicine, Teikyo University School of Medicine, Tokyo
}

Key words: airway inflammation, cytokines, airway remod-
eling

\section{Introduction}

Chronic obstructive pulmonary disease (COPD) and bronchial asthma (asthma) are characterized by obstructive pulmonary disorder. Reversibility of airflow limitation characteristically exists in asthma but not in COPD. These diseases often coexist in some patients, who are diagnosed as "asthma associated with COPD" or "COPD with asthma" (1). I will overview the recent understanding of COPD and asthma.

\section{COPD}

Definition

COPD is a disease induced by either chronic bronchitis or chronic pulmonary emphysema, or both. COPD is mostly seen in smokers; it is slowly progressive and irreversible. Chronic bronchitis is diagnosed by the clinical symptom, i.e. productive cough continuing for more than 3 months per year for more than 2 consecutive years. On the contrary, chronic pulmonary emphysema is diagnosed by the pathological findings, i.e. dilatation and destruction of the region distal to the terminal bronchioles without obvious fibrosis.

\section{Pathogenesis}

Attention has been attracted by the hypothesis regarding the

Reprint requests should be addressed to Dr. Ken Ohta, Professor of Medicine, Department of Medicine, Teikyo University School of Medicine, 2-11-1 Kaga, Itabashi-ku, Tokyo 173-8606 
Molecular Pathogenesis and Clinical Affairs of Obstructive Lung Disease, Especially Focusing on Chronic Obstructive Pulmonary Disease (COPD) and Bronchial Asthma

imbalance between elastase and anti-elastase. The hypothesis was proposed based on studies of $\alpha 1$-antitrypsin deficiency. The major source of elastase is suspected to be neutrophils and alveolar macrophages, which upon activation release too much elastase to be inactivated by anti-elastase. Smoking has been thought to play an important role to activate the cells; also air pollutants and infection with bacteria or virus may contribute to exacerbations in COPD. But it is important to remember that only $15 \%$ of smokers suffer from COPD, suggesting that some genetic factors in addition to smoking may be involved in the pathogenesis of COPD.

In 1960 Orie et al. described "the Dutch hypothesis", in which allergy and airway hyperresponsiveness are important as constitutional factors. However, the results of further investigation have rather denied at least the involvement of allergy.

\section{Molecular pathogenesis}

In COPD, neutrophils and alveolar macrophages play a central role by releasing proteinase on activation by smoking to destruct the lung tissue. Moreover, eosinophils are also seen at the lung tissue although their number is not so prominent as in the case of asthma. T cells accumulated at the pulmonary lesion are involved in the inflammatory response via cytokine production. $T$ cells accumulated at the pulmonary lesion are both $\mathrm{CD} 4$ and $\mathrm{CD} 8$ positive cells (2). Interestingly, CD4 positive cells consist of more Th1 type cells than Th2 type cells, which is opposite to the pattern seen in asthma. Investigation with induced sputum revealed an increase of interleukin-8 (IL8 ) and tumor necrosis factor- $\alpha(\mathrm{TNF}-\alpha)$ (3). IL- 8 can induce activation and migration of neutrophils and TNF- $\alpha$ can activate various cells and upregulate their cytokine synthesis and expression of adhesion molecules, suggesting that these cytokines are involved in neutrophilic as well as eosinophilic inflammation. Another cytokine presumably important is granulocyte-macrophage colony-stimulating factor (GM-CSF), which can activate neutrophils, eosinophils and macrophages, and is produced by various cells including $\mathrm{T}$ cells, airway epithelial cells and vascular endothelial cells. Research in the field of COPD to clarify its pathogenesis at the molecular level has not been fully developed, and further investigation is obviously needed.

\section{Clinical affairs}

As aforementioned, the pathogenesis of COPD has not been clarified yet, and the treatment for COPD has not been well established except for smoking cessation which may slow down the progression of the disease and oxygen therapy which may improve the survival period. Use of corticosteroids known to possess strong antiinflammatory action has been under investigation, but the results have been disappointing so far (4). Further elucidation of the pathogenesis of COPD will facilitate determination of a new therapeutic strategy to establish effective medication with newly developed agents.

\section{Bronchial asthma \\ Definition}

Asthma is a disease (or a syndrome) characterized by airway inflammation and airflow limitation with various severity levels, and appears with attacks of cough, wheeze and dyspnea. Usually the airflow limitation in asthma is reversible, but its reversibility could be decreased by airway remodeling.

\section{Pathogenesis}

About $70 \%$ of asthmatic patients have IgE antibodies to housedust mites, suggesting that allergic reaction plays an important role in the induction of airway inflammation and asthmatic symptoms. However, the mechanism through which asthma is induced in those whose allergen is not identified without involving an allergic reaction is not known. Induction of airway inflammation results in airway hyperresponsiveness and develops airway remodeling to repair the injured airway. Airway inflammation itself can be a cause of airflow limitation, and asthmatic symptoms appear when some stimuli act on the hyperresponsive airway to constrict the airway smooth muscle. Genetic factors could be involved in each of these components.

\section{Molecular pathogenesis}

\section{1) Airway inflammation}

Airway inflammation in asthma has been characterized by three features as follows; (1) desquamation of airway epithelium, (2) thickening of the region adjacent to the basement membrane, and (3) accumulation of eosinophils.

We have established a murine asthmatic model by immunizing mice with ovalbumin (OA) to raise anti-OA IgE antibodies and exposing them to the immunized antigen, i.e. OA, repeatedly to induce airway inflammation comparable with the three characteristic features of human asthmatic airways as mentioned above. By using anti-GM-CSF neutralizing antibody in our murine model, we have found that GM-CSF plays a pivotal role in the pathogenesis of asthma (5). Similar investigations by other groups have revealed that $\mathrm{Th} 2$ cytokines such as IL-4, IL-5 and IL-13 are intimately involved in the pathogenesis of asthma.

The importance of airway inflammation in asthma has been shown by further investigation with our OA-sensitized murine model. Briefly, we induced apoptosis in the inflammatory cells, mostly eosinophils, by giving anti-Fas antibody. As results, we observed disappearance of airway inflammation and inhibition of airway hyperresponsiveness (unpublished data; reported at the 2000 Annual Meeting of American Thoracic Society).

\section{2) Airway remodeling}

Airway remodeling occurs as a process of repair from injury. The process of repair for the injured airway hypothetically consists of three steps, i.e. (1)migration of airway epithelial cells (AEC) capable of differentiation such as basal cells, goblet cells and Clara cells to the region where cilliated cells are desquamated, (2) proliferation of the migrated AEC, and (3) differentiation of the proliferating AEC into cilliated epithelial cells. Insulin-like growth factor-I (IGF-I) and fibronectin are involved in migration of AEC ( (1) ). Additionally, trans- 
forming growth factor- $\beta$ (TGF- $\beta$ ) accelerates the process of migration via autocrine and paracrine. IGF-I, TGF- $\alpha$ and keratinocyte growth factor (KGF) are involved in proliferation ( (2) ), which is suppressed by TGF- $\beta$. In cellular differentiation (3), TGF- $\beta$ and retinoids play important roles in the presence of physiological airflow to complete the repair. We have clarified the pivotal roles of TGF- $\beta$ and IGF-I in our OA-sensitized murine model (unpublished data; reported at the 2000 Annual Meeting of American Thoracic Society).

3) Genetic factors

It will become realistic to perform "tailor-made medicine" in the future if the molecular pathogenesis in each patient can be clarified and genes governing drug sensitivity can also be identified.

\section{Clinical affairs}

Asthma is assumed to be a repetition of the dual asthmatic response induced by antigen exposure. Therefore, it is highly beneficial to avoid exposure to the allergen. Use of inhaled corticosteroids is very effective medication $(4,6)$. The agents act via various pharmacological effects to suppress production of inflammatory cytokines as well as the function of eosinophils and lymphocytes, and to enhance formation of anti-inflammatory substances and expression of $\beta$-adrenergic receptors. There are new therapeutic strategies which could be prom- ising such as inhibition of Th2 cytokines, i.e. for IL-4 with soluble IL-4 receptor and for IL-5 with an antibody against IL5 , and inhibition of IgE-mediated reaction with an antibody against the binding site of IgE to the IgE receptor. Clinical trials with these agents are underway in other countries, and the initial results have just been reported. We look forward to hearing their outcome for the development of new strategies to treat asthma.

\section{References}

1) American Thoracic Society. Standards for the diagnosis and care of patients with chronic obstructive pulmonary disease. Am Rev Respir Dis 152: S78-S83, 1995.

2) Jeffery PK. Structural and inflammatory changes in COPD: A comparison with asthma. Thorax 53: 129-136, 1998.

3) Keatings VM, Collins PD, Scott DM, Barnes PJ. Differences in interleukin8 and tumor necrosis factor- $\alpha$ in induced sputum from patients with chronic obstructive pulmonary disease or asthma. Am J Respir Crit Care Med 153: 530-534, 1996.

4) Keatings VM, Jatakanon A, Worsdel Y-M, Barnes PJ. Effect of inhaled and oral glucocorticoids on inflammatory indices in asthma and COPD. Am J Respir Crit Care Med 155: 542-548, 1997.

5) Ohta K, Yamashita N, Tajima M, et al. Diesel exhaust particulate induces airway hyperresponsiveness in a murine model: Essential role of GMCSF. J Allergy Clin Immunol 104: 1024-1030, 1999.

6) Ohta K, Yamashita N. Apoptosis of eosinophils and lymphocytes in allergic inflammation. J Allergy Clin Immunol 104: 14-21, 1999.

\title{
3. Immunological Lung Disease -Recent Advances in the Pathogenesis of Hypersensitivity Pneumonitis
}

\author{
Yasuyuki Yoshizawa, Tadashi Furuie, Yoshio Otani, Yuki Sumi, Megumi Sawada, \\ Takeshi Umino, Naohiko InASE and Shuji MiYake
}

Tokyo Medical and Dental University, The Pulmonary Medicine, Tokyo $\begin{array}{ll}\text { Keywards: } & \text { summer-type hypersensitivity pneumonitis, pi- } \\ & \text { geon breeder's disease, Th1 cell line, IL-10 }\end{array}$

Hypersensitivity pneumonitis (HP) is an immunologicallyinduced lung disease that develops after inhalation of various antigens. The immunologic mechanisms involved in the pathogenesis of HP have been postulated to be due to an initial immune complex-mediated lung injury, followed by cell-mediated tissue damage (1).

Several lines of recent observations have contributed to the understanding of the pathogenesis of HP leading to clinical applications for disease management.

\section{Characterization and purification of responsible antigen com- ponents for the disease}

Responsible antigen components for summer-type HP

Summer-type HP is the most prevalent HP in Japan, comprising greater than $74 \%$ of all HP. Recently, Trichosporon asahii and mucoides have been identified as the causative agents. Mizobe et al have characterized the responsible antigenic components as glucuronoxylomannan which consists of a $(1,3)$-linked mannan backbone attached to short side chains 